\title{
Experiment and Numerical Simulation of Peculiarities in the Development of Helium DC Discharge in Reflex Geometry
}

\author{
Alexandr SAMOKHIN, Andrey GAVRIKOV, Gennadii LIZIAKIN, \\ Ravil USMANOV and Valentin SMIRNOV \\ Joint Institute for High Temperatures of the Russian Academy of Sciences, Moscow, Russia
}

(Received 18 May 2016 / Accepted 24 August 2016)

\begin{abstract}
Reflex discharge has a large number of applications. However, there is no analytical formula allowing for the specified geometry to calculate the current or spatial characteristics of the discharge a priori. To do this, we create two-component model of the discharge in a diffusion-drift approximation in a magnetic field. The conditions of breakdown have been investigated in the framework of the Townsend mechanism. Current-voltage (I-V) characteristics, temporal variation of the discharge current and average ion density, and spatial distributions of ions and electrons have been calculated. I-V discharge characteristics in the absence of magnetic field have been measured. Experiments have been carried out in the discharge with a cylindrical anode with a length of $180 \mathrm{~cm}$, diameter of $90 \mathrm{~cm}$ and a flat circular cathode with a diameter of $4 \mathrm{~cm}$. The dependence of discharge current on the applied magnetic field has been determined. The dependence of steady-state discharge current on the order of electric and magnetic field switching has been studied. Comparison of theory with experiment provides qualitative agreement and it confirms the adequacy of the created model.
\end{abstract}

(c) 2016 The Japan Society of Plasma Science and Nuclear Fusion Research

Keywords: gas discharge, reflex discharge, penning discharge, diffusion-drift approximation, magnetic field

DOI: $10.1585 /$ pfr. 11.1401116

\section{Introduction}

Investigations of reflex discharge have been used for the creation of high-current nonrelativistic electron beams, in particular, those employed for plasma modification of surfaces [1]. In this case, the discharge forms plasma channel, which allows the space charge of electrons to be compensated and a high-current beam to be transported to the target surface. Reflex discharge can also be used in investigations of the electrostatic potential formation [2] and controlled motion of ions [3,4] in plasma. Earlier published data on the reflex discharge were exhaustively reviewed in [5].

The present work was aimed at (i) numerical simulation of the temporal evolution and spatial characteristics of discharge in the framework of a drift-diffusion model and (ii) experimental verification of the results of calculations. Distinctive features of the system under consideration were the inhomogeneity of electric field and extremal values of field strength at the cathode $E / P \sim 10^{3} \div$ $10^{4} \mathrm{~V} /(\mathrm{cm} \cdot$ Torr) [6].

Investigation of the conditions of electric breakdown in gaseous media is an important tool for the characterization of elementary processes in electric field in plasma volume and on the cathode surface. For helium, a relationship between the breakdown voltage and product $P d$ of the gas pressure and interelectrode distance (Paschen's curve) in the region of minimum voltage (Paschen's mini- mum at $P d \sim 4$ Torr $\cdot \mathrm{cm}$ ) represents a double-valued zigzag function $[7,8]$. A competition between electrons and fast ions with energies $\sim 1 \mathrm{keV}$ for ionization events accounts for a complicated shape of the Paschen curve [8]. At small interelectrode distances comparable with the ionization length, an important issue is establishing the equilibrium value of the Townsend ionization coefficient for the primary avalanche of electrons emitted from the cathode surface $[7,9]$. Numerical simulation of discharge in helium by the particle method at a field strength of $E / P=$ $5 \cdot 10^{4} \mathrm{~V} /(\mathrm{cm} \cdot$ Torr $)$ confirmed the Townsend breakdown mechanism [7], showed it to be valid for any $E / P$ provided that $P d>0.17$ Torr $\cdot \mathrm{cm}$, and allowed an expression for the Townsend coefficient to be obtained by approximating the numerical data.

The influence of magnetic field on the electric breakdown in a cylindrical diode was recently experimentally studied in [10]. Transport coefficients of charged-particle swarms in gases under the influence of electric and magnetic fields crossed at arbitrary angles were calculated in [11]. Discharge in crossed electric and magnetic fields obeys the principle of equivalent pressure with respect to the ionization rate [12] and the principle of equivalent reducedelectric field for the Townsend ionization coefficient $[13,14]$. The influence of transverse magnetic field on the second Townsend coefficient and the effective electrode gap principle related to confinement of the transverse size of electron avalanche by discharge tube walls were 


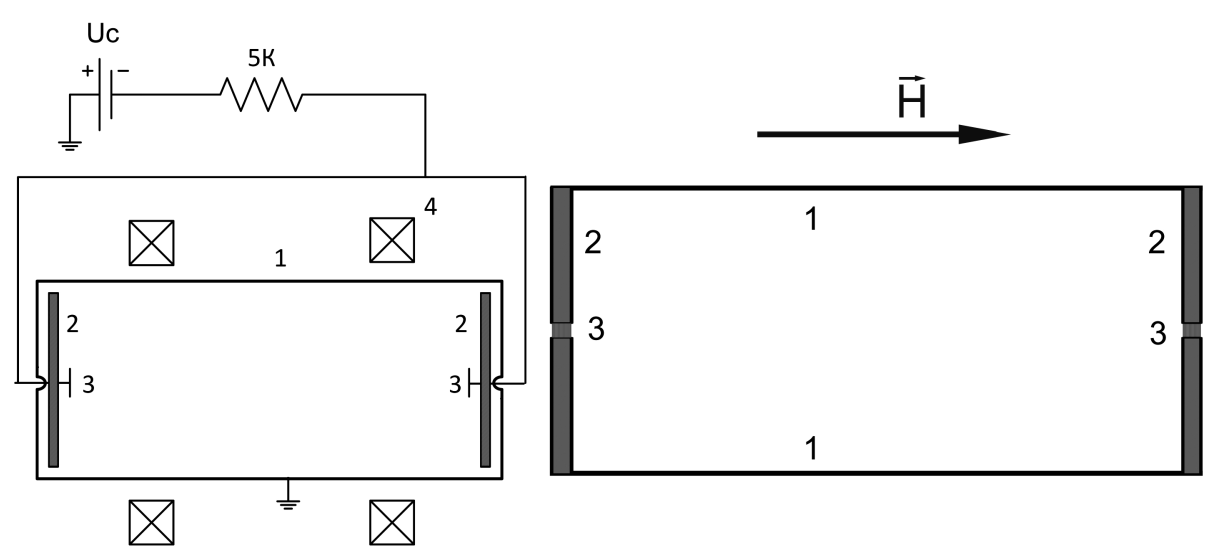

(a)

(b)

Fig. 1 Schematic diagram of the experimental setup (a) and computational domain (b) (see text for explanations).

considered in $[15,16]$. The present investigation follows the approach outlined by Blevin and Haydon [12].

\section{Experimental Setup and Computa- tional Domain}

Figure 1 shows a schematic diagram of the experimental setup. Grounded cylindrical vacuum chamber 1 with a length of $200 \mathrm{~cm}$ and a diameter of $90 \mathrm{~cm}$ served as the anode of discharge. Dielectric disks 2 screened the chamber edges from the discharge gap. Two identical cathodes 3 with diameter of $4 \mathrm{~cm}$ were mounted on the dielectric plates and positioned exactly opposite to each other on the cylinder axis. The distance between cathodes was $180 \mathrm{~cm}$. Both cathodes and the anode were made of stainless steel. The magnetic field was created by coils 4 . The coils have inner diameter of $100 \mathrm{~cm}$, and they stand at a distance of $50 \mathrm{~cm}$ from each other.

The computational domain has some differences from experimental setup. First, we use homogeneous magnetic field for simulation. Second, dielectric ends of domain were close to the anode. Third, the cathodes will not stick out from dielectric ends.

\section{Criterion of Breakdown in Inhomo- geneous Electric Field}

\subsection{Breakdown in the absence of magnetic field}

For constructing a model of discharge, it is necessary to determine the effective coefficient of electron multiplication on the cathode. In order to find this coefficient in the framework of the Townsend theory, it is sufficient to measure the breakdown voltage for a given geometry of discharge and electrode materials. According to [7], the Townsend theory is valid for discharge in helium provided that $P d>0.17$ Torr $\cdot \mathrm{cm}$ (i.e., for interelectrode gap widths greater that the characteristic length of electron multiplica- tion). In the case under consideration with a helium pressure of $35 \mathrm{mTorr}$, this condition is satisfied. At voltages below $1 \mathrm{kV}$, the ion-induced electron emission coefficient is $\gamma \approx 0.016(I-2 \varphi)=0.25$ for helium ions, where $I$ is the ionization potential and $\varphi[\mathrm{eV}]$ is the work function (for the potential emission mechanism [17]).

According to the Townsend theory, the criterion $\eta$ of breakdown in inhomogeneous field $E(s)$ can be expressed as follows [6]:

$$
\begin{aligned}
& \eta=\frac{P}{\ln (1+1 / \gamma)} \int_{0}^{s_{A}} \alpha_{\mathrm{T}}(E(s) / P) \mathrm{d} s>1, \\
& \alpha_{\mathrm{T}}(E / P) \\
& \quad=5.4 \cdot \exp (-14 \sqrt{P / E}-0.0017 \cdot(E / P))
\end{aligned}
$$

Here, $\alpha_{\mathrm{T}}\left[\mathrm{cm}^{-1}\right.$. Torr $\left.{ }^{-1}\right]=\alpha / P$ is the Townsend ionization coefficient for helium, reduced to a pressure of $P=$ 1 Torr [7]; $\gamma$ is effective ion-induced electron emission coefficient (second Townsend coefficient); $s$ is the coordinate measured along the electron trajectory from cathode to anode; and $S_{A}$ is the total trajectory length. An important property of the adopted Townsend coefficient is that $\alpha_{\mathrm{T}}$ is a nonmonotonic function with maximum at $E / P=$ $257 \mathrm{~V} /(\mathrm{cm} \cdot$ Torr), which corresponds (for $P=35 \mathrm{mTorr}$ ) to $E_{0}=9.0 \mathrm{~V} / \mathrm{cm}$.

The computational region of the cylindrical discharge chamber $(r<R=45 \mathrm{~cm}, 0<z<L=200 \mathrm{~cm})$ was bounded in the radial direction by the conducting cylinder (anode) and in the direction of axis $Z$ by dielectric walls with flat round central electrodes (cathodes) with $r<R_{\mathrm{c}}=2 \mathrm{~cm}$. The electric field $E(s)$ at any given voltage $U$ was calculated using the differential Laplace equation with boundary conditions

$$
\begin{gathered}
\left.\phi\right|_{r=R}=0,\left.\quad \phi\right|_{r<R_{\mathrm{c}}, z=0}=-U \\
\left.(\partial \phi / \partial r)\right|_{r=0}=\left.(\partial \phi / \partial z)\right|_{z=0.5 L} \\
=\left.(\partial \phi / \partial z)\right|_{r>R_{\mathrm{c}}, z=0, L}=0 .
\end{gathered}
$$



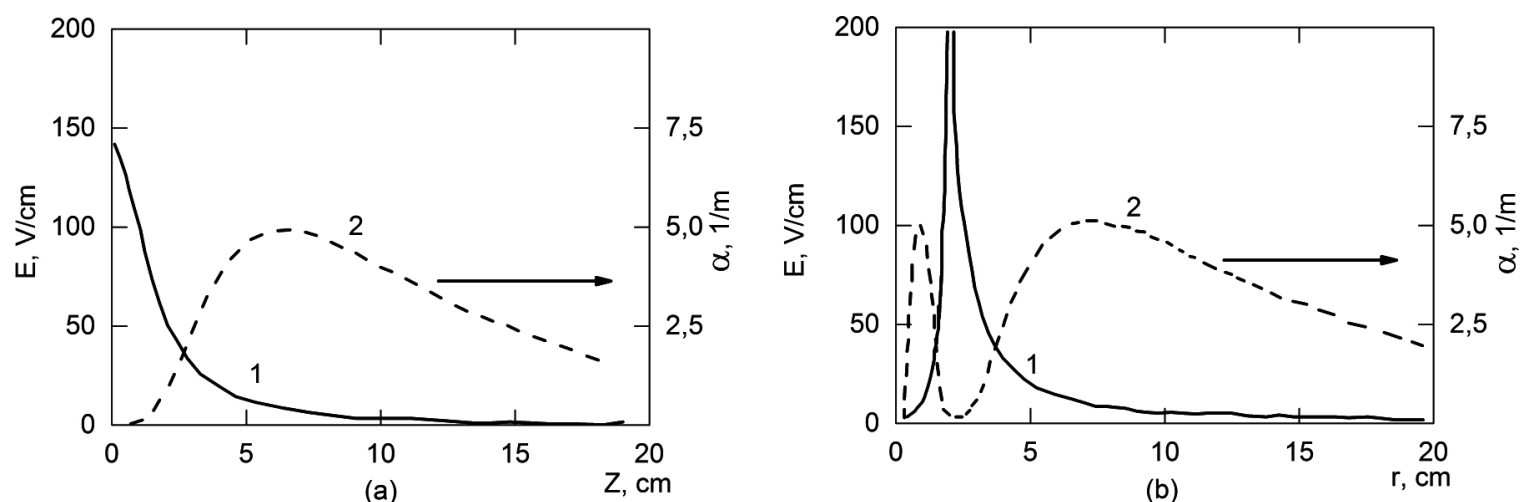

Fig. 2 Electric field strength $E_{z}, E_{r}(\mathbf{1})$, and Townsend ionization coefficient $\alpha(\mathbf{2})$ : (a) along axis $Z(r=0)$; (b) in radial direction (in plane $z=0.20 \mathrm{~cm}$ ). Helium pressure, $P=35 \mathrm{mTorr}$; discharge voltage, $U=400 \mathrm{~V}$.

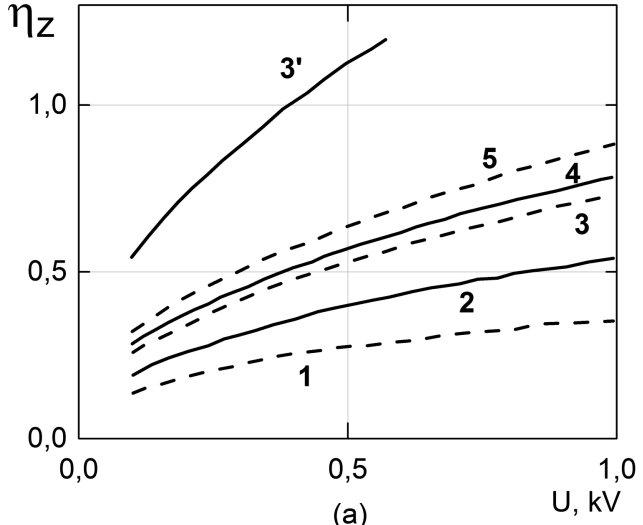

(a)

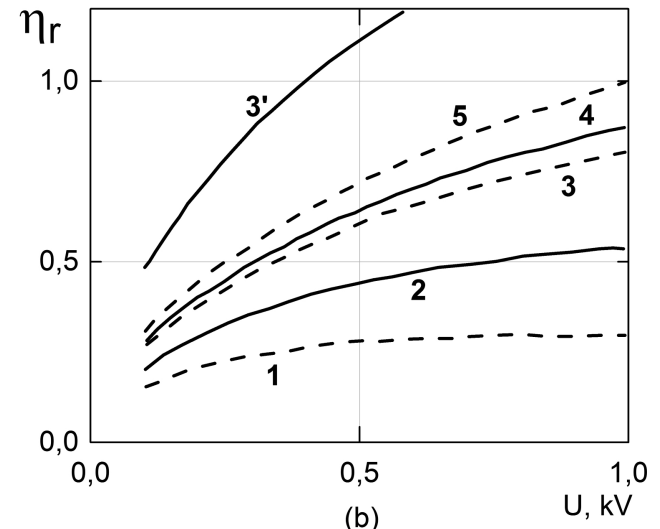

(b)

Fig. 3 Breakdown parameters $\eta_{z}$ and $\eta_{r}$ vs. applied voltage (a) along axis $Z$ and (b) in radial direction $(z=0.20 \mathrm{~cm})$ for helium pressures $P[\mathrm{mTorr}]=10(\mathbf{1}), 20(\mathbf{2}), 35(\mathbf{3}), 40(\mathbf{4})$, and $50(\mathbf{5})$ at $\gamma=0.25$ and for $P=35 \mathrm{mTorr}\left(\mathbf{3}^{\prime}\right)$ at $\gamma=0.89$ (a) and 0.73 (b).

Here, the second line corresponds to the conditions of symmetry on the chamber axis, in the central plane $(z=$ $100 \mathrm{~cm}$ ), and on the dielectric edge plane. Calculations were performed on a rectangular inhomogeneous grid with a minimum step near the central electrode $(\Delta z=0.20 \mathrm{~cm}$, $\Delta r=0.19 \mathrm{~cm}$ ). Figures 2 (a) and 2 (b) show the obtained distributions of the electric field strength and Townsend ionization coefficient along axis $Z$ and in the radial direction near the edge plane (at $z=0.20 \mathrm{~cm}$ ).

The inhomogeneity of electric field, which is caused by the central flat cathode with radius $R_{\mathrm{c}}=2 \mathrm{~cm}$, significantly restricts the spatial scale on which a local equilibrium between electrons and electric field has to be established. In the axial direction, the minimum characteristic size of this inhomogeneity $[\mathrm{d} \ln (E) / \mathrm{d} z]^{-1} \sim 5 \mathrm{~cm}$ is observed immediately at the electrode surface $(z=0)$, while the maximum Townsend coefficient is attained at $z=7 \mathrm{~cm}$ (Fig. 2(a)). The efficiency of ionization is determined by the integral of the Townsend coefficient over axial coordinate $z$, which is almost independent of the near-electrode region. Analogous considerations are valid for the electric field distribution in the radial direction (Fig. 2(b)). A small difference between the dependences of the Townsend coefficient on coordinates $z$ and $r$ is due to small relative di- mensions of the cathode: $2 R_{\mathrm{c}} / L=0.02$ and $R_{\mathrm{c}} / R=0.044$. To the first approximation, the electric field strength (similar to that of a point charge) is inversely proportional to certain power $f(1<f<2)$ of the distance from cathode. The position of maximum of the ionization coefficient corresponds to $E_{\mathrm{o}}=9.0 \mathrm{~V} / \mathrm{cm}$ and is situated rather far from the electrode (at intermediate distance between minimum and maximum dimensions of the system).

A comparison of the conditions of breakdown in the radial direction near a chamber edge (in the plane $z=$ $0.20 \mathrm{~cm})$ and along the chamber axis $(r=0)$ shows that, at a helium pressure above 35 mTorr with neglect of plasma recombination on the dielectric wall, the discharge is more readily initiated in radial direction near the edge (Fig. 3). The experiment showed that the breakdown between anode and cathode takes place at $400 \mathrm{~V}$. Therefore, we select those values of $\gamma$ with which $\eta_{z}$ and $\eta_{r}$ can reach 1 at $400 \mathrm{~V}$. For $\eta_{z}$ the value of $\gamma$ is 0.89 and for $\eta_{r}-\gamma=0.73$.

\subsection{Breakdown in the presence of magnetic field}

In order to model the dependence of the ionization coefficient on the electric field in the axial magnetic field, let 

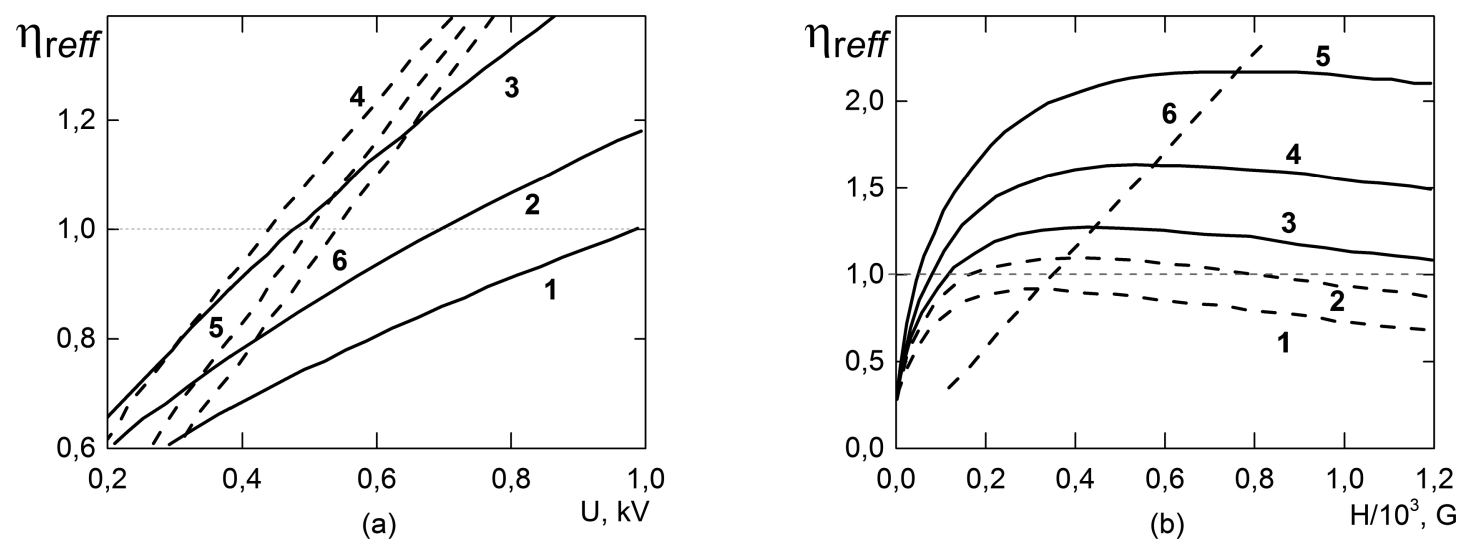

Fig. 4 Breakdown parameter $\eta_{r \text { eff }}$ in the radial direction in plane $z=0.2 \mathrm{~cm}$ in inhomogeneous electric and magnetic fields: (a) vs. $U$ at $H / 10^{3}[\mathrm{G}]=0.07(\mathbf{1}), 0.1(\mathbf{2}), 0.2(\mathbf{3}), 0.4(\mathbf{4}), 0.8(\mathbf{5})$, and $1(\mathbf{6})$; (b) vs. $H$ at $U[\mathrm{kV}]=0.5(\mathbf{1}), 0.6(\mathbf{2}), 0.7(\mathbf{3}), 0.9(\mathbf{4}), 1.2(\mathbf{5})$; dash-dot line (6) shows maximum $\eta_{r \text { eff }}$ for $\gamma=0.01, P=35$ mTorr, $v_{\mathrm{e}}=0.88 \cdot 10^{8} \mathrm{~s}^{-1}$.

us introduce the notion of effective ionization rate in magnetic field [12] and define the effective field strength $E_{\text {eff }}$ as the radial field component $E_{r}$ divided by factor $\sqrt{1+\chi_{\mathrm{e}}^{2}}$ :

$$
\begin{aligned}
& v_{\mathrm{eff}}=\mu_{\mathrm{e} 0} E_{\mathrm{eff}} \alpha_{\mathrm{T}}\left(E_{\mathrm{eff}} / P\right), \\
& E_{\mathrm{eff}}=\sqrt{E_{z}^{2}+E_{r}^{2} /\left(1+\chi_{\mathrm{e}}^{2}\right),} \\
& \chi_{\mathrm{e}}=\omega_{\mathrm{Ce}} / v_{\mathrm{e}} .
\end{aligned}
$$

Here, $v_{\mathrm{eff}}$ is the effective ionization rate; $\omega_{\mathrm{Ce}}$ and $v_{\mathrm{e}}$ are the cyclotron frequency and transport frequency of electron collisions with helium atoms and ions, respectively; $\mu_{\mathrm{e} 0}\left[\right.$ Torr $\left.\cdot \mathrm{cm}^{2} / \mathrm{V} \cdot \mathrm{s}\right]=\mu_{\mathrm{e}} P$ is the electron mobility reduced to a pressure of 1 Torr; and $\chi_{\mathrm{e}}$ is the Hall parameter [18]. In contrast to earlier investigations of discharge in a transverse magnetic field $[13,15,16]$, where the effective field strength $E_{\text {eff }}$ was used to determine the Townsend coefficient $\alpha_{\mathrm{T}}$, relation (3) defines the effective ionization rate in magnetic field [12]. The effective breakdown condition and the corresponding Townsend coefficient are as follows:

$$
\begin{aligned}
\eta_{\mathrm{eff}} & =\frac{P}{\ln (1+1 / \gamma)} \int_{0}^{S_{A}} \alpha_{\mathrm{Teff}}\left(E(s)_{\mathrm{eff}} / P\right) \mathrm{d} s>1, \\
\alpha_{\mathrm{Teff}} & =v_{\mathrm{eff}} / \mu_{\mathrm{e} 0} \sqrt{E_{z}^{2}+\left[E_{r} /\left(1+\chi_{\mathrm{e}}^{2}\right)\right]^{2}} \\
& =\sqrt{\frac{E_{z}^{2}+E_{r}^{2} /\left(1+\chi_{\mathrm{e}}^{2}\right)}{E_{z}^{2}+\left[E_{r} /\left(1+\chi_{\mathrm{e}}^{2}\right)\right]^{2}}} \alpha_{\mathrm{T}}\left(E_{\mathrm{eff}} / P\right) .
\end{aligned}
$$

The value of $\alpha_{\mathrm{T} \text { eff }}$ can be greater than $\alpha_{\mathrm{T}}$ for two reasons. First, because the first term in expression for $\alpha_{\mathrm{T} \text { eff }}$ at $E_{z}=0$ is $\sqrt{1+\chi_{\mathrm{e}}^{2}}>1$, which reflects an increase in the electron trajectory length as a result of the drift in crossed electric and magnetic fields in the azimuthal direction. Another reason is a decrease in $\alpha_{\mathrm{T}}$ with increasing field strength at large values of the argument, since the replacement of $E$ by smaller $E_{\mathrm{eff}}$ leads to an increase in the ionization coefficient.

Figure 4 shows calculated values of the effective breakdown parameter $\eta_{r \text { eff }}$ in the radial direction in plane $z=0.20 \mathrm{~cm}$. At fixed $H$, an increase in $U$ within $0 \div 1 \mathrm{kV}$ leads to a growth in $\eta_{r \text { eff }}$ (Fig. 4 (a)). In a magnetic field of $70 \mathrm{G}$, the condition of breakdown $\eta_{r \text { eff }}>1$ is attained at a voltage of $U \geq 1 \mathrm{kV}$ (Fig. 4 (a), curve 1). At fixed $U$, the dependence of $\eta_{r \text { eff }}$ on magnetic field $H$ is nonmonotonic (Fig. 4(b)). For $\gamma=0.01$ and $U=600 \mathrm{~V}$, the breakdown is possible in the interval of magnetic fields $H$ from 180 to $800 \mathrm{G}$ (Fig. 4 (b), curve 2), while a greater voltages this interval expands predominantly toward stronger magnetic fields.

\section{Drift-Diffusion Approximation for Discharge in Helium in the Pres- ence of Magnetic Field}

Two-component plasma of electrons and singly charged ions in magnetic field has been numerically simulated in the framework of a drift-diffusion approximation [18] by solving the Poisson's equation for potential $\varphi$,

$$
-\Delta \phi=4 \pi e\left(n_{\mathrm{i}}-n_{\mathrm{e}}\right),
$$

and the system of equations for electron concentration $n_{\mathrm{e}}$ and ion concentration $n_{\mathrm{i}}$,

$$
\begin{array}{ll}
\frac{\partial n_{\mathrm{e}}}{\partial t}+\operatorname{div} \Gamma_{\mathrm{e}}=\dot{q}_{\mathrm{e}}, & \Gamma_{\mathrm{e}}=-\left(\mu_{\mathrm{e}} \bar{E}+D_{\mathrm{e}} \bar{\nabla}\right) n_{\mathrm{e}}, \\
\frac{\partial n_{\mathrm{i}}}{\partial t}+\operatorname{div} \Gamma_{\mathrm{i}}=\dot{q}_{\mathrm{i}}, & \Gamma_{\mathrm{i}}=\left(\mu_{\mathrm{i}} \bar{E}-D_{\mathrm{i}} \bar{\nabla}\right) n_{\mathrm{i}} .
\end{array}
$$

Mobilities $\mu_{\mathrm{e}}, \mu_{\mathrm{i}}$ can be expressed via the corresponding collision frequencies $v_{\mathrm{e}}, v_{\mathrm{i}}$ and the Hall parameters $\chi_{\mathrm{e}}$, $\chi_{\mathrm{i}}$ as

$$
\begin{aligned}
& \mu_{\mathrm{e}}=\frac{e}{m_{\mathrm{e}} v_{\mathrm{e}}} M\left(\chi_{\mathrm{e}}\right), \quad \mu_{\mathrm{i}}=\frac{e}{A m_{H} v_{\mathrm{i}}} M\left(\chi_{\mathrm{i}}\right), \\
& M(\chi)=\left(\begin{array}{ccc}
\frac{1}{1+\chi^{2}} & \frac{\chi}{1+\chi^{2}} & 0 \\
\frac{-\chi}{1+\chi^{2}} & \frac{1}{1+\chi^{2}} & 0 \\
0 & 0 & 1
\end{array}\right),
\end{aligned}
$$




$$
\begin{aligned}
& \chi_{\mathrm{e}}=\omega_{\mathrm{Be}} / \nu_{\mathrm{e}}, \quad \omega_{\mathrm{Be}}[1 / \mathrm{s}]=1.7 \cdot 10^{7} H[\mathrm{G}], \\
& \chi_{\mathrm{i}}=\omega_{\mathrm{Bi}} / v_{\mathrm{i}}, \quad \omega_{\mathrm{Bi}}[1 / \mathrm{s}]=0.96 \cdot 10^{4} A^{-1} H[\mathrm{G}],
\end{aligned}
$$

where $A=4$ is the atomic mass of helium ion. The diffusion tensor is assumed to be proportional to the mobility (Einstein's relationship) [6]:

$$
D_{a}\left[\mathrm{~cm}^{2} / \mathrm{s}\right]=T_{a}[\mathrm{eV}] \mu_{a}\left[\mathrm{~cm}^{2} / \mathrm{s} \times \mathrm{V}\right], a=e, i .
$$

The rates of charge production in the magnetic field are $\dot{q}_{\mathrm{i}}=\dot{q}_{\mathrm{e}}=v_{\mathrm{eff}} \cdot n_{\mathrm{e}}$, where the effective ionization rate $v_{\mathrm{eff}}$ (see Eq. (3)) in the magnetic field is determined by the effective field strength $E_{\text {eff }}$ that depends on the applied electric field $E$ and Hall parameter $\chi_{\mathrm{e}}$.

Equation (5) has to be supplemented by the boundary condition for potential $\varphi$, which were formulated above for determining the electric field in vacuum (see Eq. (2), where the condition of symmetry on the edge is replaced by the condition of charge equilibrium on the dielectric):

$$
\left.\left(\Gamma_{\mathrm{i}}-\Gamma_{\mathrm{e}}\right)_{z}\right|_{r>R_{\mathrm{c}} z=0}=0 .
$$

Boundary conditions for the system of Eqs. (6) include, in addition to the conditions of symmetry on axis $Z$, in the central plane, and on the edge plane (analogous to those for the potential), the following conditions on the electrodes and dielectric:

$$
\begin{aligned}
& \left.\left(\gamma \Gamma_{\mathrm{i}}-\Gamma_{\mathrm{e}}\right)_{z}\right|_{r<R_{\mathrm{c}}, z=0}=0 \text { (on cathode); } \\
& \left.n_{\mathrm{i}}\right|_{r=R}=0 \\
& \left.\left(\partial n_{\mathrm{e}} / \partial r\right)\right|_{r=R}=0 \text { (on anode); } \\
& \left.\left(\partial n_{\mathrm{i}} / \partial z\right)\right|_{r>R_{\mathrm{c}}, z=0}=\left.\left(\partial n_{\mathrm{e}} / \partial z\right)\right|_{r>R_{\mathrm{c}}, z=0} \\
& \quad=0 \text { (on dielectric) } .
\end{aligned}
$$

We neglect the inhomogeneity of the electron density near the anode i.e. electron flow to the anode is the flow of electrons with thermal velocity.

\section{Discharge without Magnetic Field 5.1 Current-voltage characteristics and sec- ondary electron yield}

Let us determine the effective coefficient of electron multiplication on the cathode of discharge chamber by using the measured the current-voltage $(I-V)$ characteristic and comparing the experimental value of the breakdown voltage to that according to the Townsend model (Fig. 3). The experiment showed that the breakdown between anode and cathode takes place at $400 \mathrm{~V}$. When the voltage between electrodes was increased in the absence of magnetic field, the discharge current at $400 \mathrm{~V}$ exhibited a jump-like growth by two orders of magnitude (from 0.1 to $10 \mu \mathrm{A}$ ). According to the results of calculations, this voltage corresponds to breakdown parameter $\eta=1$ for $\gamma=0.89$ in the direction of axis $Z$ and for $\gamma=0.73$ in the radial direction. Figure 5 compares the experimental $I-U$ curve to the characteristic calculated using Eqs. (5) and (6) for

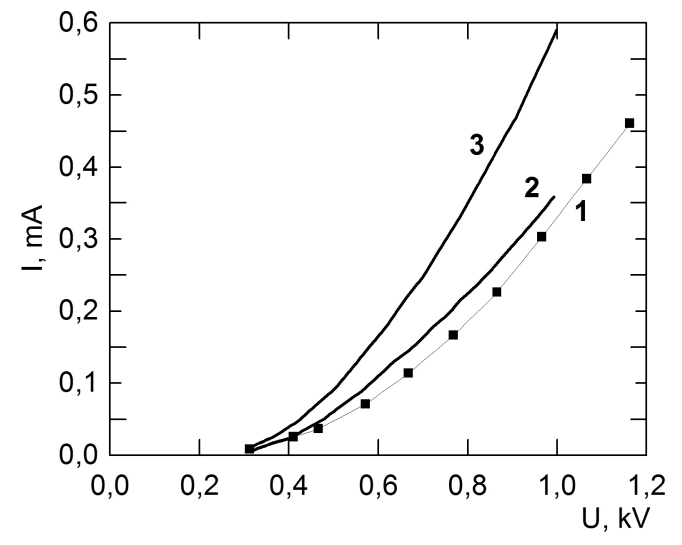

Fig. 5 Current-voltage characteristics of discharge: (1) experiment; $(2, \mathbf{3})$ calculation for $T_{\mathrm{e}}=4$ and $10 \mathrm{eV}$, respectively. Gas pressure $35 \mathrm{mTorr}, \gamma=1$.

$\gamma=1$ at electron temperatures $T_{\mathrm{e}}=4$ and $10 \mathrm{eV}$. At a discharge voltage of $0.9 \mathrm{kV}$, the plasma potential measured in the central plane on the chamber axis $(r=0, z=100 \mathrm{~cm})$ amounts to $\sim 4 \mathrm{~V}$, which coincides with the value of plasma potential calculated in simulation. This simulation was carried out with assumption that an electron temperature was $4 \mathrm{eV}$.

A difference between $\gamma=0.25$ adopted in literature and the value of $\gamma \sim 1$ that follows from the comparison with experiment implies that the integral of $\alpha_{\mathrm{T}}(s) \mathrm{d} s$ calculated in the present work over the longest electron drift trajectory in the chamber is smaller by a factor of $\ln (5) / \ln (2)=2.3$. Probably, there exists a trajectory intermediate between the longest and shortest one (lying in the edge plane), for which the corresponding integral takes the maximum value that is sufficient to obey the Townsend criterion at the lower value of $\gamma=0.25$.

In what follows, the numerical simulations have been performed for a base value of $\gamma=1$ and it is implied that $\gamma$ is an effective coefficient of proportionality between the flux of ions and the flux of emitted electrons, which sums up all physical processes taking place in the near-electrode space.

\subsection{Numerical simulation of discharge}

Discharge in a cylindrical region $(0<r<R, 0<z<$ $Z$ ) corresponding to half of the discharge chamber has been numerically simulated for the following initial profile of plasma density distribution with maximum at the cathode center:

$$
n_{\mathrm{e}}=n_{\mathrm{i}}=10^{4} \cos ^{4}(\pi r / 2 R) \cos ^{2}(\pi z / 2 Z),
$$

where $R=45 \mathrm{~cm}, Z=100 \mathrm{~cm}$, and the average number of particles in the chamber is

$$
<n_{\mathrm{e}, \mathrm{i}}>=0.86 \cdot 10^{3} \mathrm{~cm}^{-3} \text {. }
$$

In the computational model, a stationary state is attained for a period of time $\sim 0.2 \mathrm{~ms}$ and corresponds to a current of $0.11 \mathrm{~mA}$ at a voltage of $U=0.6 \mathrm{kV}$ and $0.36 \mathrm{~mA}$ 

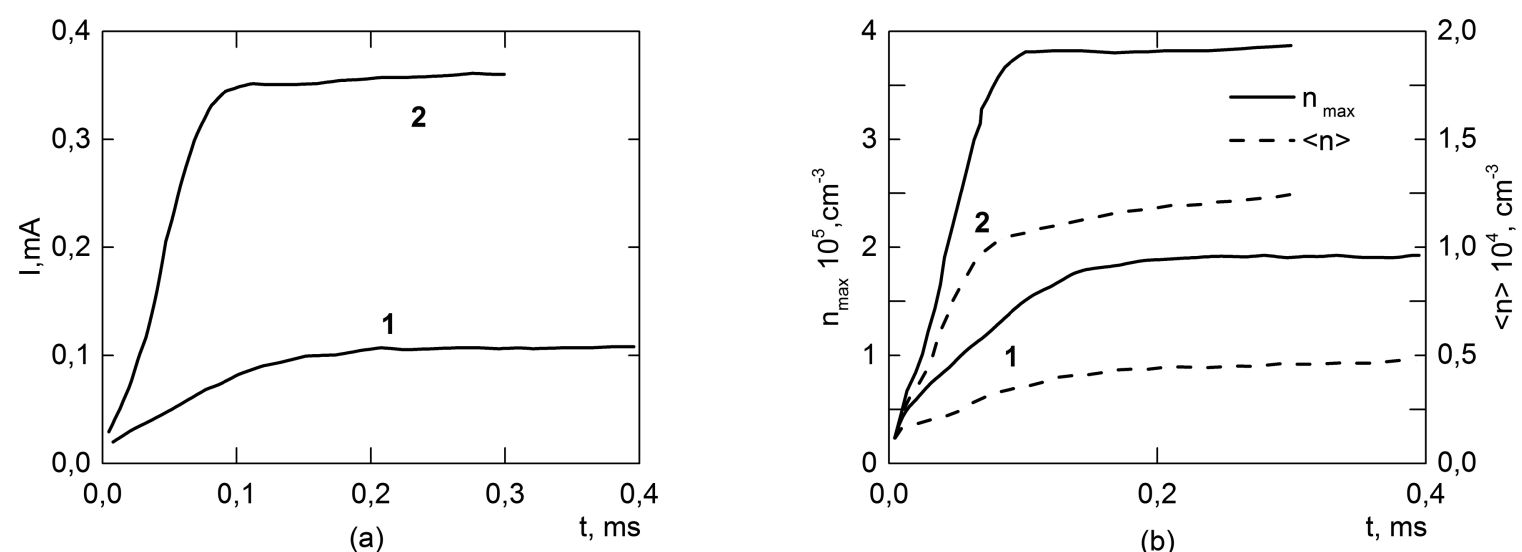

Fig. 6 Temporal variation of (a) the ion current and (b) concentration; $T_{\mathrm{e}}=4 \mathrm{eV}, U=0.6 \mathrm{kV}$ (1) and $1 \mathrm{kV}$ (2).
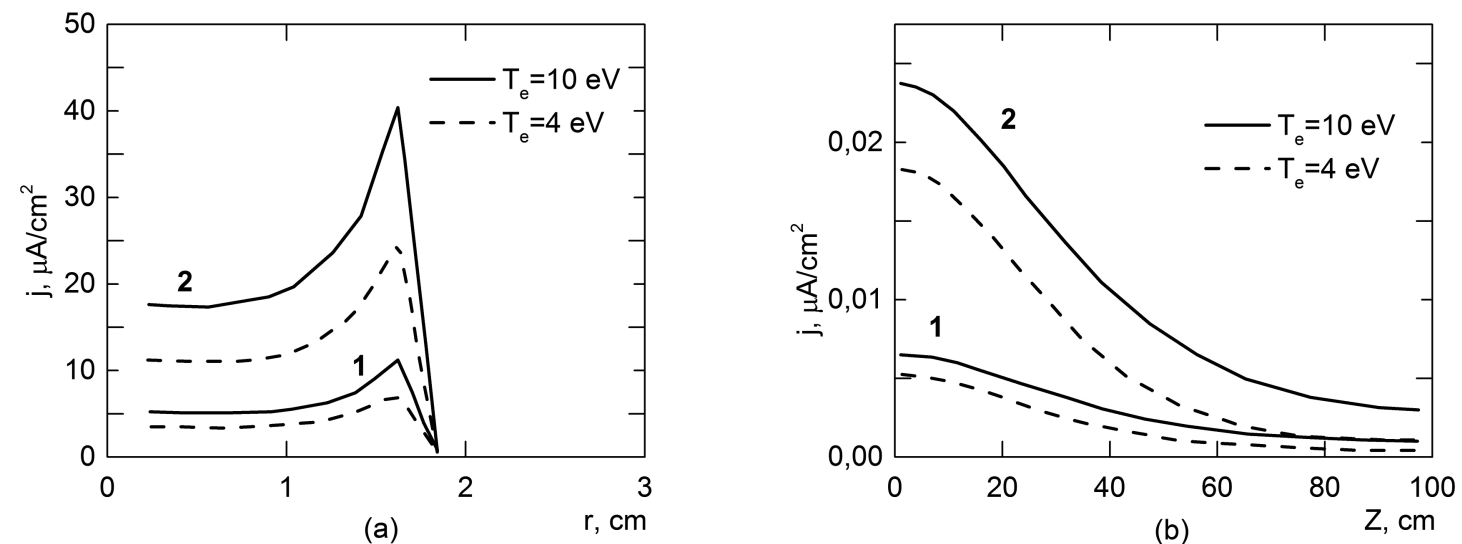

Fig. 7 Distribution of current density over (a) cathode surface and (b) anode surface for $U=0.6 \mathrm{kV}(\mathbf{1})$ and $1 \mathrm{kV}(\mathbf{2})$.
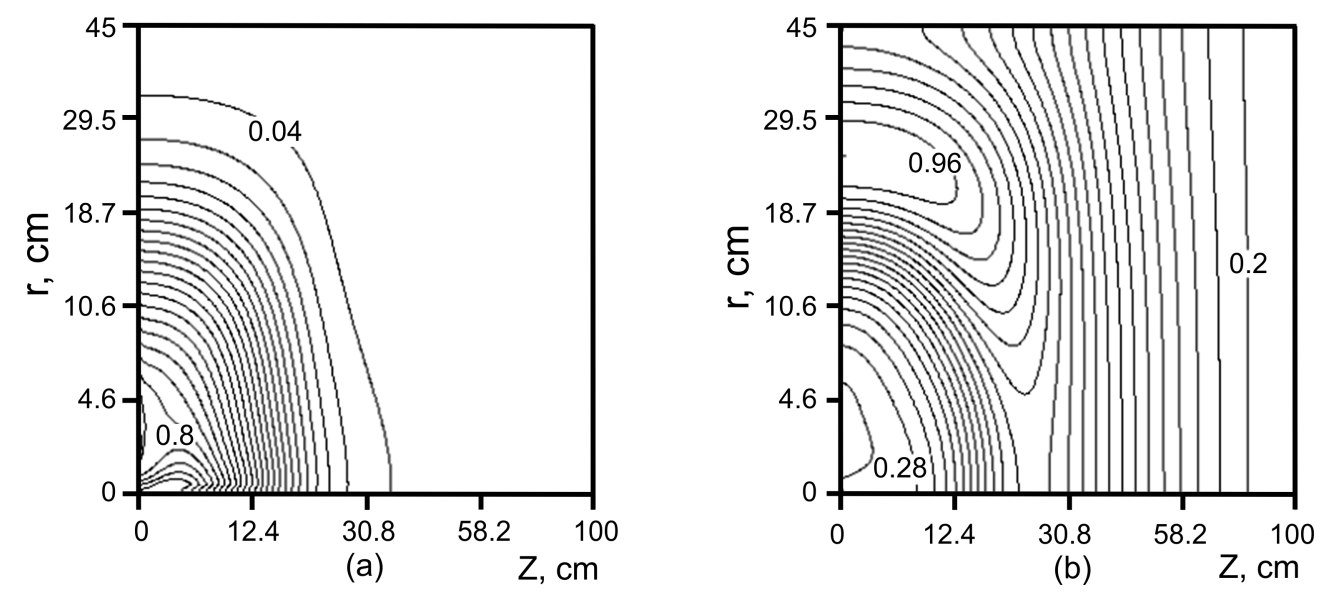

Fig. 8 Spatial distributions of (a) ion concentration $n_{\mathrm{i}} / n_{\mathrm{i}, \max }\left(n_{\mathrm{i}, \max }=3 \cdot 10^{5} \mathrm{~cm}^{-3}\right)$ and (b) electron concentration $n_{\mathrm{e}} / n_{\mathrm{e}, \max }\left(n_{\mathrm{e}, \max }=\right.$ $\left.6 \cdot 10^{4} \mathrm{~cm}^{-3}\right) ; T_{\mathrm{e}}=4 \mathrm{eV}, U=0.6 \mathrm{kV}$.

at $U=1 \mathrm{kV}$ (Fig. 6). Figure 6 shows the temporal variation of ion current and the average and maximum ion density, which confirm the attainment of a stationary state. The charge equilibrium manifested by the equality of currents to cathode and anode is established much faster than the equilibrium state of discharge as the whole. The distribution of current over the surface of electrodes (Fig. 7) and the distribution of particles in the volume (Fig. 8) indicate that the current passes predominantly in the radial direction near the edge $(0<z<40 \mathrm{~cm})$.

Boundary effects are strongly pronounced on the cathode, where the maximum current density at the periphery is about two times as large as that at the cathode center. Plasma density in the volume is not large, and the concentrations of electrons and ions differ by one order of magnitude (Fig. 8). 

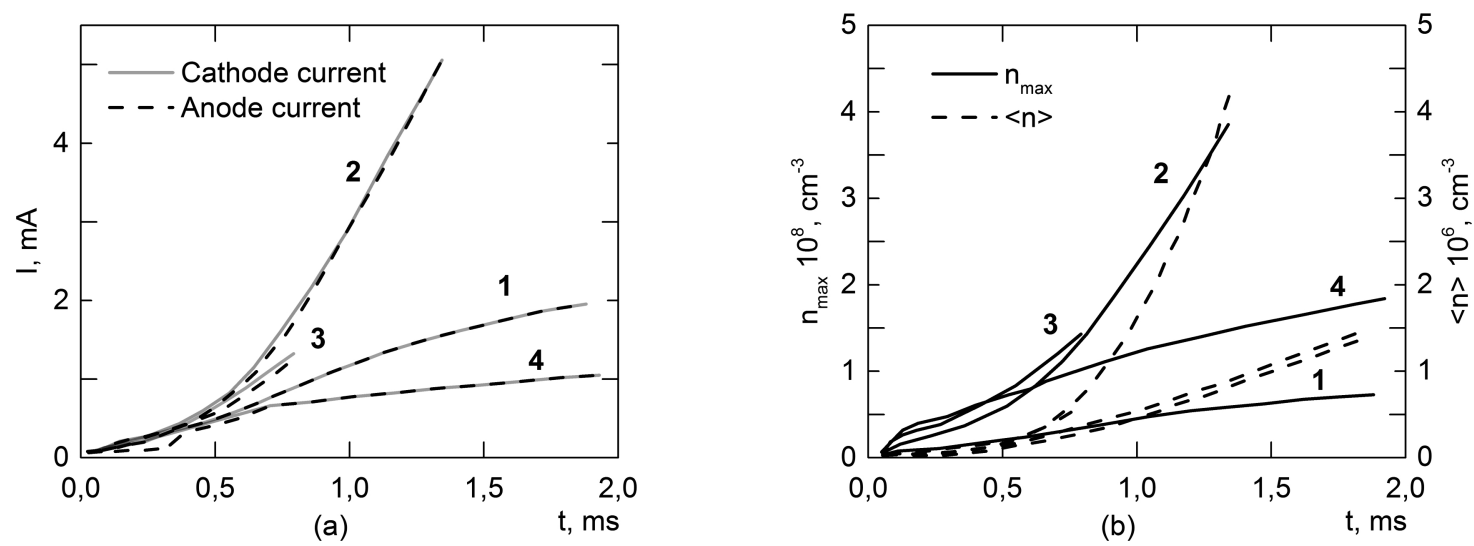

Fig. 9 Temporal variation of (a) the ion current and (b) concentration; $H[\mathrm{G}]=100(\mathbf{1}), 200(\mathbf{2}), 300(\mathbf{3})$, and $400(\mathbf{4}) ; T_{\mathrm{e}}=4 \mathrm{eV}$, $U=0.6 \mathrm{kV}$.
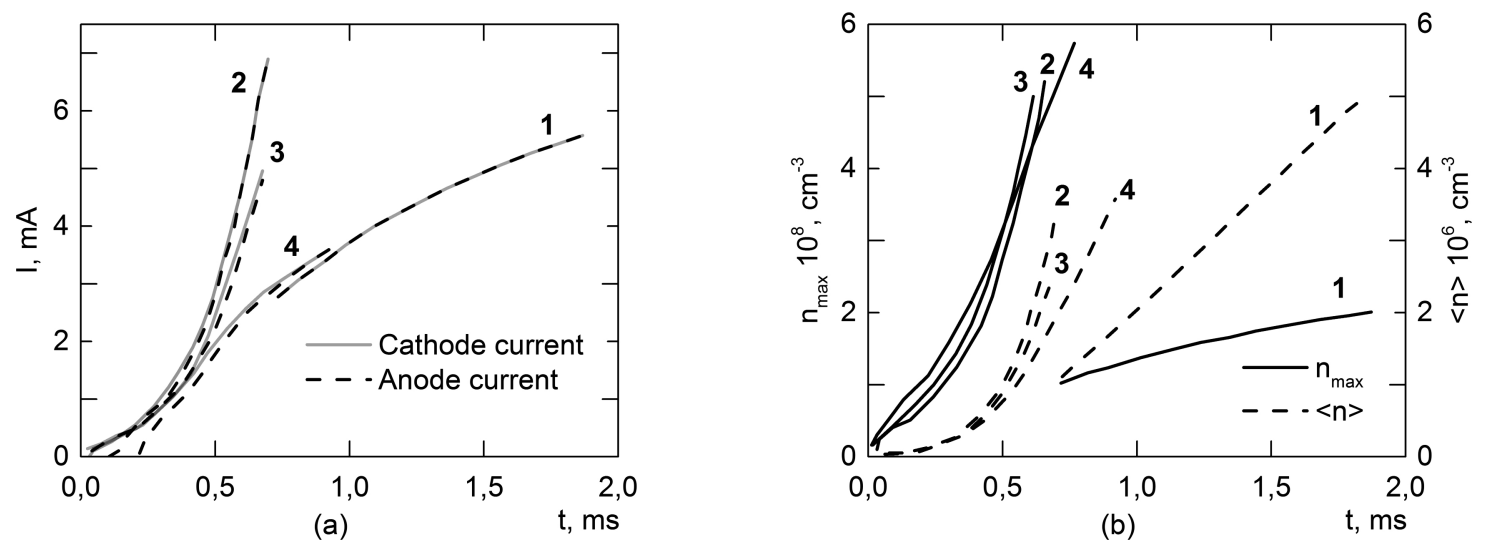

Fig. 10 Temporal variation of the ion current (a) and concentration (b); $H[\mathrm{G}]=100(\mathbf{1}), 200(\mathbf{2}), 300(\mathbf{3})$, and $400(\mathbf{4}) ; T_{\mathrm{e}}=4 \mathrm{eV}$, $U=1 \mathrm{kV}$.

\section{Discharge in Magnetic Field}

\subsection{Numerical simulation of discharge}

In contrast to the case of discharge without magnetic field considered above, calculations over a period of time $t$ necessary for the establishment of a stationary state in the presence of magnetic field were difficult to carry out because of a strongly increased plasma concentration and restricted time step $\Delta t$.

With initial conditions for the plasma density used above in the case of $H=0$, simulations carried out at a voltage of $0.6 \mathrm{kV}$ in magnetic field $H=100 \mathrm{G}$ show an increase in the ion current and concentration within $\sim 2 \mathrm{~ms}$ and pronounced tendency of discharge current stabilization on a level of $\sim 2 \mathrm{~mA}$ (Fig. 9 (a)). For the same discharge voltage in magnetic field $H=200 \mathrm{G}$, the current reaches $5.1 \mathrm{~mA}$ for a shorter period and continues linear growth with the time. In a field of $H=300 \mathrm{G}$, the current increases slower than in the case of $H=200 \mathrm{G}$ but still faster than at $100 \mathrm{G}$. In a still stronger magnetic field of $H=400 \mathrm{G}$, the derivative $\mathrm{d} I / \mathrm{d} t$ decreases to minimum and the current reaches a stationary value of $1 \mathrm{~mA}$ for $2 \mathrm{~ms}$. An analogous behavior is observed for $U=1 \mathrm{kV}$ (Fig. 10 (a)).
The results of numerical simulations show a nonmonotonic dependence of current on the magnetic field, with a maximum current attained for $H \sim 200 \mathrm{G}$.

Temporal variation of the maximum and volumeaveraged ion concentrations in magnetic field is more complicated than that in the case of $H=0$. In magnetic fields $H=100$ and $400 \mathrm{G}$, the growth rate of maximum plasma density begins to decrease simultaneously with the time derivative of the discharge current. At the same time, the average ion concentration continues to grow at a constant rate (Fig. 9(b)) over the entire computational period of $2 \mathrm{~ms}$. At a voltage of $U=600 \mathrm{~V}$, the current and maximum ion concentration are $2 \mathrm{~mA}$ and $0.72 \cdot 10^{8} \mathrm{~cm}^{-3}$ in a field of $100 \mathrm{G}$ and $1 \mathrm{~mA}$ and $1.85 \cdot 10^{8} \mathrm{~cm}^{-3}$ in a field of $400 \mathrm{G}$ (i.e., a greater current corresponds to lower ion concentration). At $U=1 \mathrm{kV}$ and almost equal currents of $3.6 \mathrm{~mA}$, the maximum density of plasma in a field of $H=400 \mathrm{G}$ is about four times that for $H=100 \mathrm{G}$ (Fig. 10 (b)).

The application of magnetic field modifies the distribution of current over the surface of electrodes (Fig. 11). The boundary effects on the cathode (with the current density reaching maximum at the periphery and being signifi- 

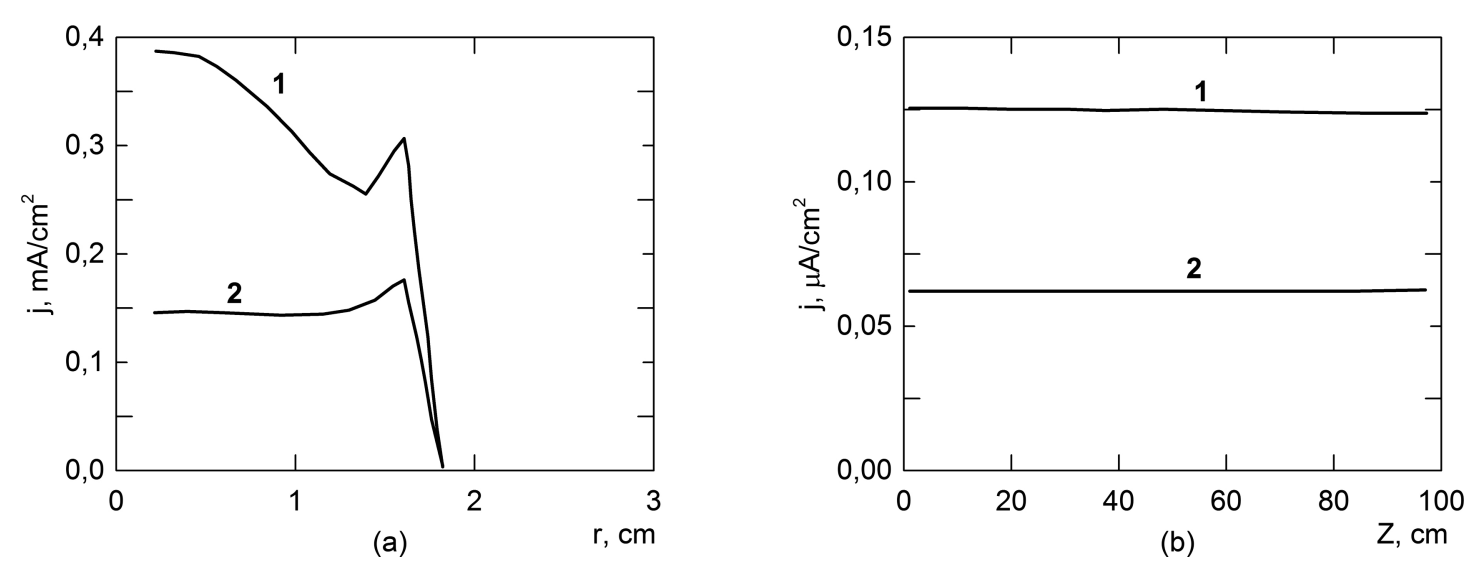

Fig. 11 Distribution of current density over (a) cathode surface and (b) anode surface for $H[\mathrm{G}]=200(\mathbf{1})$ and $400(\mathbf{2}) ; T_{\mathrm{e}}=4 \mathrm{eV}$, $U=1 \mathrm{kV}$.
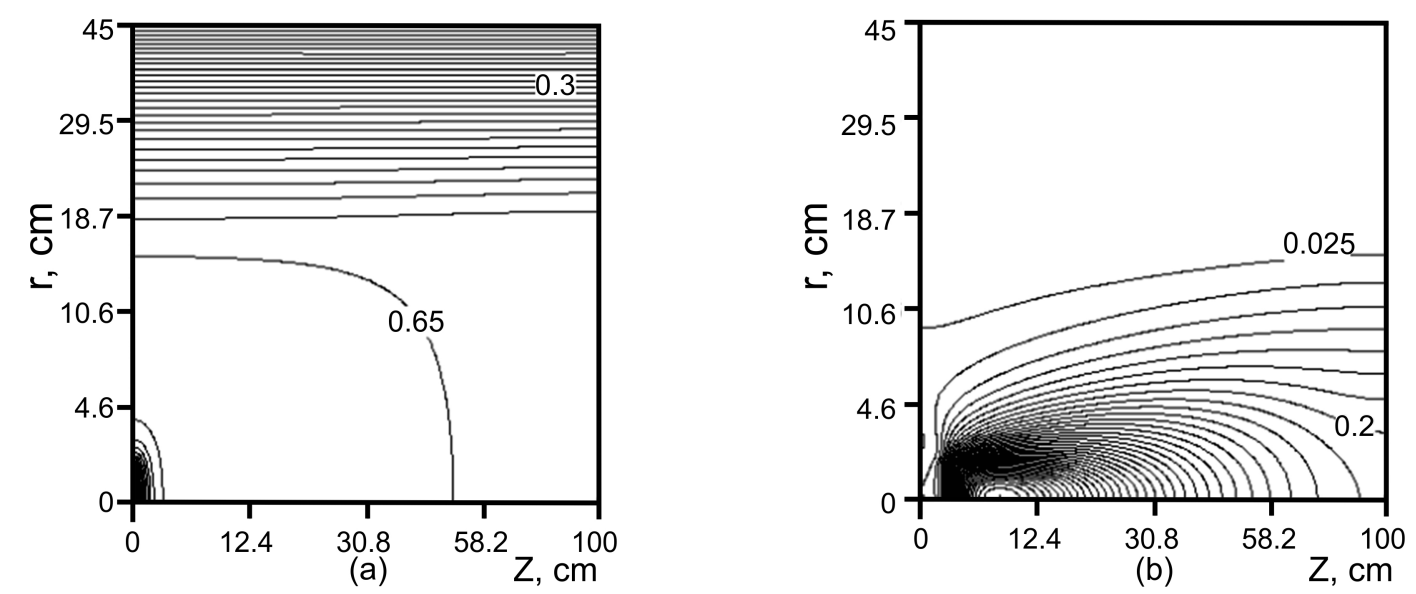

Fig. 12 Spatial distributions of (a) electric field $\varphi / U$ and (b) ion concentration $n_{\mathrm{i}} / n_{\mathrm{i} \max }\left(n_{\mathrm{i} \max }=3.8 \cdot 10^{8} \mathrm{~cm}^{-3}\right)$ in discharge at $T_{\mathrm{e}}=4 \mathrm{eV}$, $H=200 \mathrm{G}, U=0.6 \mathrm{kV}, t=1.34 \mathrm{~ms}$. Curves represent equilevel lines at $0.025 \mathrm{step}$.

cantly greater than at the center) is more pronounced in the absence of magnetic field (Fig. 7(a)). In discharge with magnetic field $H=200 \mathrm{G}$, the current density exhibits maximum on the axis ( $r=0$, Fig. 11 (a)). In contrast to the case of $H=0$, the discharge in magnetic field is characterized by a constant current density on the outer coaxial electrode (Fig. 11 (b)).

The spatial distributions of electric field and ion concentration in discharge with magnetic field (Fig. 12) are qualitatively different from the distributions of field and plasma density in the absence of magnetic field. Because of a strong anisotropy of conductivity in the magnetic field, the equipotential surfaces of electric field are aligned along axis $Z$ (Fig. 12 (a)) outside a paraxial region of $r>15 \mathrm{~cm}$ in the chamber. Plasma is concentrated at the axis of chamber in front of the cathode (Fig. 12 (b)). There is a qualitative agreement between the pattern of calculated equilevel lines of ion concentration and the picture of glow on the photographs of discharge (Fig. 13).

\subsection{Experimental observations and discus- sion}

It was established that the discharge current depends

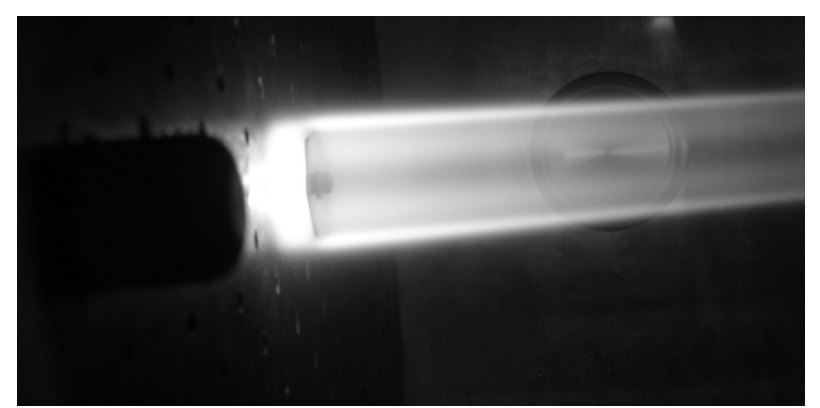

Fig. 13 Photograph of discharge at $I=40 \mathrm{~mA}, U=660 \mathrm{~V}, H=$ $840 \mathrm{G}$.

not only on the applied voltage and magnetic field, but also on the order of switching of the electric and magnetic fields. This is probably related to the fact that the stationary discharge current is significantly influenced by the primary breakdown process that is substantially different for the developement of discharge with and without magnetic field. Figure 14 shows the plots of the experimentally measured discharge current versus magnetic field. In measuring the dependences presented in Fig. 14 (a), the magnetic field was switched on first and then the voltage was ap- 


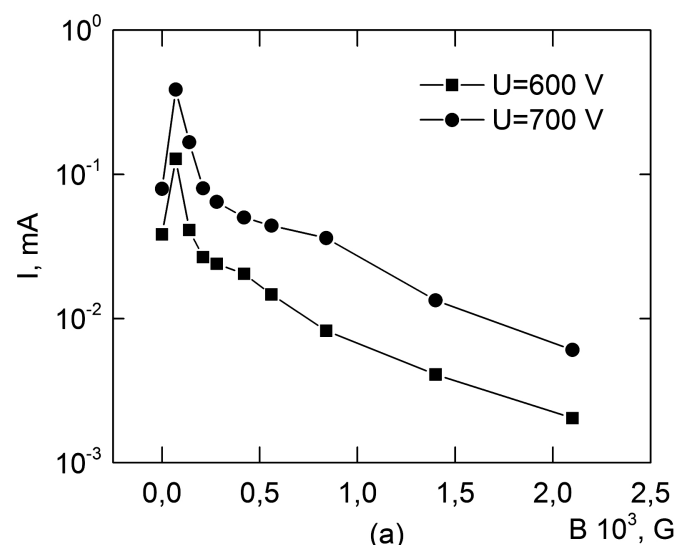

(a)

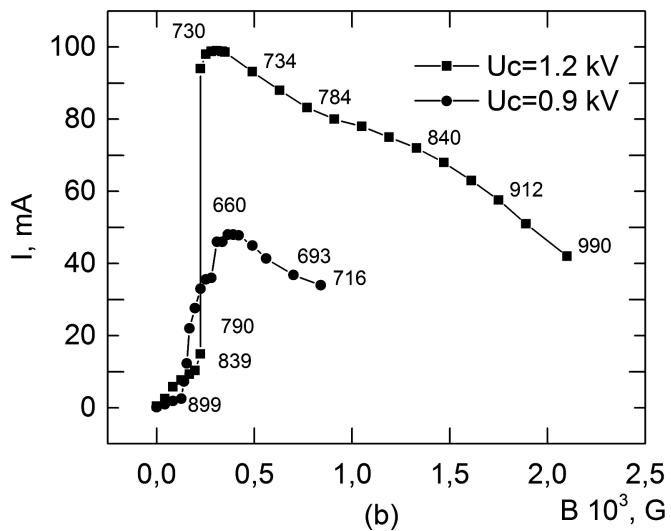

(b)

Fig. 14 Dependence of the discharge current on magnetic field: (a) low-current discharge mode (b) high-current discharge mode. Interelectrode voltage are indicated at the points.

plied. If the fields were applied in the reverse order, the mode of discharge at some parameters exhibited a qualitative change. As can be seen in Fig. 14 (b), the application of initial voltage $U=1200 \mathrm{~V}$ followed by gradual increase in the magnetic field up to $220 \mathrm{G}$ is accompanied by a linear increase in the discharge current. However, already in a field of $230 \mathrm{G}$, the current exhibits a tenfold growth (from 10 to $100 \mathrm{~mA}$ ), so that the discharge passes to a highcurrent mode.

The experimental dependences of current $I$ on magnetic field $H$ exhibit three peculiarities. The first is the nonmonotonic character of this dependence with a maximum at $H=100 \mathrm{G}$ for applied voltages $U=600$ and $700 \mathrm{~V}$ (Fig. 14 (a)). The second feature is a significant increase in the current at $H=225 \mathrm{G}$ for $U_{\mathrm{C}}=1200 \mathrm{~V}$ and at $H=140 \mathrm{G}$ for $U_{\mathrm{C}}=900 \mathrm{~V}$ (Fig. $14(\mathrm{~b})$ ). $U_{\mathrm{C}}$ is a power supply voltage applied to the circuit with a ballast resistor $R_{\mathrm{C}}=5 \mathrm{kOhm}$ (not the discharge voltage). An analogous dependence was reported in [19]. The third feature is the existence of low-current $(\sim 0.1 \mathrm{~mA})$ and high-current $(\sim 100 \mathrm{~mA})$ modes of discharge.

The differential relationship:

$$
\begin{gathered}
-\left(R_{\mathrm{C}}+\partial U(I, H) / \partial I\right) \cdot \mathrm{d} I / \mathrm{d} H \\
=\partial U(I, H) / \partial H,
\end{gathered}
$$

observed in the case of $R_{\mathrm{C}}=0$ (Fig. 14 (a)) implies that $\partial U / \partial H=0$ for $H=100 \mathrm{G}$. Assuming that the $I-U$ characteristic measured in the interval of $0-700 \mathrm{~V}$ is increasing function so that $\partial U / \partial I>0$, we may conclude that $\partial U / \partial H<0$ for small magnetic fields $(H<100 \mathrm{G})$ in the region where $\mathrm{d} I / \mathrm{d} H$ is positive. In large magnetic fields $(H>100 \mathrm{G})$, the derivative is positive $(\partial U / \partial H>0)$ so that a growth in $H$ at a fixed current increases the resistance of discharge. A change in the sign of derivative $\partial U / \partial H$ at $H=100 \mathrm{G}$ and the nonmonotonic of $U$ on the magnetic field at a given current is analogous to the dependence of calculated breakdown parameter $\eta_{r \text { eff }}$ on the $U$, $H$ pair (Fig. 4). In a magnetic field at which the discharge passes to a high-current mode (Fig. 14 (b)), the effective breakdown parameter according to calculations (see Fig. 4) reaches $80 \%$ of the maximum value for both $U_{\mathrm{C}}=1200$ and $900 \mathrm{~V}$.

\section{Conclusion}

We have considered a model of DC discharge in the drift-diffusion approximation for two-component plasma, which takes into account the existence of nearelectrode zones and ionization processes with nonmonotonic Townsend coefficient. The $I-U$ characteristic calculated in the framework of the adopted model well agrees with the experimental dependence of the discharge current on the applied voltage.

A procedure of determining the conditions of Townsend breakdown in inhomogeneous electric field in vacuum with and without magnetic field is described. The effective electric field strength $E_{\text {eff }}$ and effective Townsend ionization coefficient $\alpha_{\mathrm{T} \text { eff }}$ have been introduced that take into account complex electron trajectories in magnetic field.

Experimental results reveal the existence of low- and high-current modes of discharge. The results of numerical calculations confirm the experimentally observed nonmonotonic dependence of current on the magnetic field with a maximum at $H=200 \mathrm{G}$ and currents above $10 \mathrm{~mA}$.

The distribution of plasma density calculated for the discharge in magnetic field agrees with the observed pattern of discharge glow with predominant concentration of plasma at the axis of discharge chamber within a radius of edge electrode (cathode).

\section{Acknowledgments}

The work was supported by a grant from the Russian Scientific Fund (project No.14-29-00231).

[1] G.E. Ozur, D.I. Proskurovsky and K.V. Karlik, Instrum. Exp. Tech. 48, 753 (2005). 
[2] A.I. Morozov, Introduction to Plasma Dynamics (CRC Press, Boca Raton, 2012).

[3] V.P. Smirnov, A.A. Samokhin, N.A. Vorona et al., Plasma Phys. Rep. 39, 456 (2013).

[4] Y.V. Kovtun, E.I. Skibenko, A.I. Skibenko et al., Tech. Phys. 56, 5, 623 (2011).

[5] E.B. Hooper Jr., Advances in Electronics and Electron Physics (L. Marton and M. Claire, Academic Press, New York, 1970) pp.295-343.

[6] Y.P. Raizer, V.I. Kisin and J.E. Allen, Gas Discharge Physics (Springer Berlin Heidelberg, 2011).

[7] A.N. Tkachev and S.I. Yakovlenko, Tech. Phys. Lett. 29, 683 (2003).

[8] A.Y. Sonin, Tech. Phys. Lett. 32, 208 (2006).

[9] D. Marić, M. Savić, J. Sivoš et al., Eur. Phys. J. D 68, 155 (2014).

[10] S.-Z. Li and H.S. Uhm, Phys. Plasmas 11, 3443 (2004).
[11] S. Dujko, R. White, Z.L. Petrović et al., Phys. Rev. E 81, 046403 (2010).

[12] H. Blevin and S. Haydon, Aust. J. Phys. 11, 18 (1958).

[13] A. Heylen and K. Bunting, Int. J. Electronics Theoretical and Experimental 27, 1 (1969).

[14] A. Heylen, IEE Proceedings A (Physical Science, Measurement and Instrumentation, Management and Education, Reviews) 127, 221 (1980).

[15] S. Sen and A. Ghosh, Proceedings of the Physical Society 80, 909 (1962).

[16] A. Heylen, Int. J. Electronics Theoretical and Experimental 41, 209 (1976).

[17] L. Dobretsov and M.V. Gomoyunova, Emission electronics (Nauka, Moscow, 1966). [in Russian]

[18] M. Mitchner and C.H. Kruger, Partially ionized gases (Wiley, New York, 1973).

[19] W. Schuurman, Physica 36, 136 (1967). 